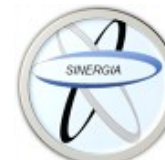

\title{
Mortalidad y perfil epidemiológico por lesiones ocupacionales en Costa Rica de 1990 al 2017
}

Mortality and epidemiologic profile of occupational injuries in Costa Rica from 1990 to 2017

\author{
${ }^{1}$ Dra. Andrea Nuñez Chaves \\ Investigadora independiente, San José, Costa Rica \\ (D) https://orcid.org/0000-0002-5643-521X \\ ${ }^{2}$ Dr. José Alberto Antúnez Oliva \\ Investigador independiente, San José, Costa Rica \\ (D) https://orcid.org/0000-0002-6776-3008 \\ ${ }^{3}$ Dr. Daniel Boza Díaz \\ Investigador independiente, Heredia, Costa Rica. \\ (D) https://orcid.org/0000-0003-1700-7367
}

Recibido
$20 / 10 / 2021$
Corregido $30 / 12 / 2021$
Aceptado $10 / 01 / 2022$

\section{RESUMEN}

Introducción. Las lesiones ocupacionales se dividen en accidentes que ocurren dentro del horario laboral, como enfermedades profesionales causadas ya sea por el tipo de actividad realizada o por el lugar en el que se desempeñan las funciones. Ha sido una de las preocupaciones más grandes a nivel mundial dentro de las regulaciones sobre Salud Ocupacional; la mortalidad ha ido en descenso, pero, aun así, en la actualidad los datos son preocupantes alrededor del mundo. Metodología. Los datos principalmente fueron obtenidos de Global Burden of Disease y de la Organización para la Cooperación y el Desarrollo Económicos. Se realizó el estudio con tasas estandarizadas y brutas la mortalidad, por lo cual todos estos datos se lograron agrupar por edad y grupo etario. Resultados. Durante el periodo en estudio, la tasa de mortalidad fue en descenso desde 1990 al 2017, la cual disminuyó de 9,51 muertes por cada 100000 personas a 4,44, siendo siempre mayor el sexo masculino entre 15 a 49 años, y la menor tasa de mortalidad se presentó para el sexo femenino, en personas mayores de 70 años. Conclusiones. La tasa de mortalidad fue mayor para el año 1990 en el sexo masculino y en personas entre 15 a 49 años; sin embargo, se evidencia descenso con respecto a los años en estudio. Finalmente, los principales problemas a nivel mundial en el ámbito laboral son: dolor de espalda (37\%), pérdida de la audición (16\%) y EPOC (13\%) y a nivel de América son: hipoacusia ocupacional, intoxicaciones por plaguicidas, metales pesados, enfermedades respiratorias y asociadas a la piel. 


\section{PALABRAS CLAVE: riesgos ocupacionales; salud ocupacional; enfermedades ocupacionales; accidentes ocupacionales; mortalidad}

\section{ABSTRACT}

Introduction. Occupational injuries are divided into accidents that occur within working hours as occupational diseases caused either by the type of activity performed or by the place where the functions are performed. It has been one of the biggest concerns worldwide in occupational health regulations, mortality has been declining, but even so, the data is currently worrying around the world. Methodology. The data was mainly obtained from the Global Burden of Disease and the Organization for Economic Cooperation and Development. The study was carried out with standardized and gross rates for the years lived with disability, years of life lost, years of life adjusted for disability, healthy years of life lost and mortality, which all these data were grouped by age and age group. Results. During the period under study, it was possible to determine that the mortality rate was in decline from 1990 to 2017, which decreased from 9.51 deaths per 100000 people to 4.44, the male sex being always between 15 and 49 years old and the lowest mortality rate for women, in older people 70 years old. Conclusions. The mortality rate was higher for the year 1990 in males and in people between 15 and 49 years, however, there is evidence of a decrease compared to the years under study

KEYWORDS: occupational risks; occupational health; occupational diseases; occupational accidents; mortality

${ }^{1}$ Médica general, graduada de la Universidad de Iberoamérica de Costa Rica (UNIBE). Cód. MED16679 . Correo: andreanunez@hotmail.com

${ }^{2}$ Médico general, graduado de la Universidad de Iberoamérica de Costa Rica (UNIBE). Cód. MED16686 . Correo: jalberto44@hotmail.com

${ }^{3}$ Médico general, graduado de la Universidad de Iberoamérica de Costa Rica (UNIBE). Cód. MED16975 . Correo: dsboza93@gmail.com

\section{INTRODUCCIÓN}

La salud ocupacional se define como: "Una concepción amplia y multidisciplinaria, encaminada a asegurar el bienestar físico, mental y social del trabajador, para lo cual, además de médico e higienista, deben contar con enfermera, psicólogo, trabajador social y ergónomo (1)"; por lo tanto, según esta definición, todos las ocupaciones, trabajos $u$ oficios deben contar con un equipo llamado Servicio de Salud Ocupacional (SSO), el cual pueda garantizar idoneidad a los servicios médicos que se brinden, cumpliendo, así, las normas legales de salud laboral y de seguridad.

Esta actividad debe llevarse a cabo en una organización u empresa como parte de la administración de recursos humanos, y debe cumplir con las normas establecidas; se intenta proveer seguridad, protección y atención a los trabajadores en el desempeño de sus actividades y funciones. De acuerdo con el tipo de trabajo o actividad que realice la empresa, es de suma importancia que ahí mismo se realice y se siga algún tipo de protocolo o manual para minimizar riesgos, y saber qué hacer en caso de emergencia, además de lograr capacitar a todo el personal.

Todas las personas, a la hora de adquirir un trabajo, tienen todo el derecho de que no les ocurra ningún accidente o lesión en el lugar o camino a su trabajo, por lo que aquí es donde se refleja la importancia de la Salud Ocupacional. Su importancia radica en intentar disminuir cualquier riesgo, daño o malestar al trabajador, manteniendo y promoviendo el bienestar de los trabajadores de manera física y 
emocionalmente. Por lo tanto, se intenta que el trabajador se sienta cómodo en el lugar donde desempeña sus funciones, para evitar consecuencias, y más bien tener buenas actitudes para una mejor calidad de trabajo. La epidemiología ocupacional es el estudio de los efectos de las exposiciones en el lugar de trabajo sobre la frecuencia, distribución de enfermedades y lesiones en la población trabajadora.

En cuanto a la salud de los trabajadores, se pueden mencionar básicamente dos problemas principales, y además se encuentran sujetos a vigilancia: los accidentes de trabajo y las enfermedades profesionales, por lo que, según la Organización Internacional del Trabajo (OIT), se estiman 250 millones de accidentes laborales cada año en todo el mundo; 3000 personas mueren a diario por causas relacionadas con el trabajo, y hay 1.1 millones de accidentes mortales en el mismo periodo (2).

La definición de accidente de trabajo es un hecho repentino relacionado casualmente con la actividad laboral, que ocasiona lesiones al trabajador o su muerte, y sus factores pueden ser por factores humanos como actos inseguros, factores técnicos como el ambiente, o factores organizativos, tanto gerenciales como administrativos (3).

Existen accidentes no mortales que pueden producir daños o lesiones de leve a mediana intensidad. Hay accidentes "in itinere" o de trayecto, que son los que ocurren en los desplazamientos desde la casa al trabajo o viceversa, y hay que tener en cuenta que si un accidente ocurre en un trabajo propio, ya sea que la casa es convertida en pequeña empresa, igualmente entra dentro de la categoría de accidente de trabajo.

Finalmente, las enfermedades de trabajo son "aquellas alteraciones de la salud nosológicamente bien definidas, producidas por la acción directa del trabajo, en trabajadores que habitualmente se exponen a factores etiológicos constantemente presentes en determinadas profesiones $u$ ocupaciones, bajo circunstancias previstas en las legislaciones respectivas (4).

\section{MÉTODO}

Este estudio es de tipo descriptivo, debido a que es una investigación observacional, el cual se toman datos y no se manipulan ni se modifican para ningún interés, sino que se reúnen para finalmente poder resolver el problema inicial, y demostrar con datos los resultados expuestos, como lo define Sampieri (5).

La metodología utilizada en este estudio de investigación fue por medio de métodos estadísticos con indicadores epidemiológicos, los cuales se lograron debido a la fórmula que se menciona a continuación (6):

Mortalidad:

$\frac{\text { cantidaddemuertesporlesionesocupacionalesenCR }}{\text { Poblaciontotallaboralmenteactiva }} \times 100000$

Se decidió calcular los datos por una población mayor de 15 años, debido a que es la población laboralmente activa en el país. Por lo tanto, luego de calcular por medio de estos indicadores, se observan los resultados y ser analizan los datos obtenidos, para crear conclusiones y recomendaciones.

\section{RESULTADOS}

Se presentará la tasa de mortalidad por lesiones ocupacionales según sexo de acuerdo al grupo etario analizado en el estudio realizado. Ver figura 1. Se observa cómo hay una marcada alta tasa de mortalidad del sexo masculino, donde se 
reporta la mayor tasa en 14,72 por cada 100 000 habitantes en 1990, en la cual, entre los 28 años en estudio, se observa una tendencia al descenso, logrando una tasa de mortalidad para este grupo en 6,86 para el 2017.
Finalmente, el grupo con menor tasa de mortalidad es para el del sexo femenino, con una tasa de mortalidad en 0,74 por cada 100000 habitantes, la cual se encuentra sin cambios significativos desde 1990 hasta el 2017.

Figura 1. Tasa de mortalidad por lesiones ocupacionales atribuibles según sexo en personas de 15 a 49 años, en Costa Rica desde 1990 al 2017 (Tasa por cada 100000 habitantes)

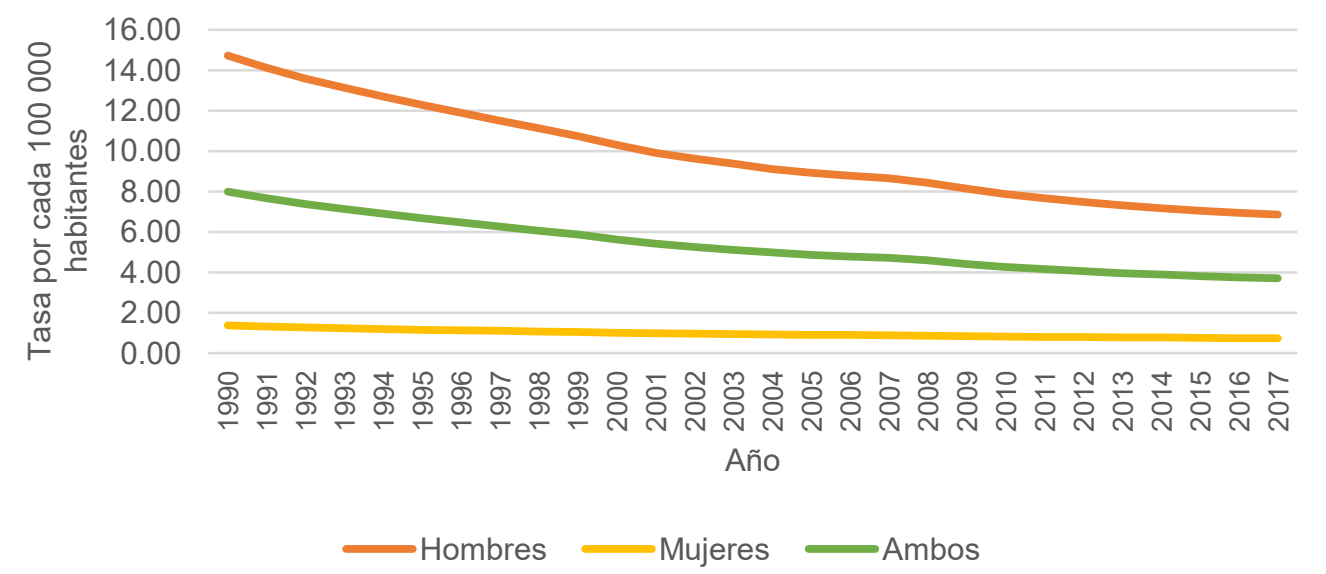

Fuente: Elaboración propia, con datos de GBD Compare | IHME Viz Hub [Internet]. [citado 19 de septiembre de 2021]. Disponible en: http://vizhub.healthdata.org/gbd-compare

Figura 2. Tasa de mortalidad por lesiones ocupacionales atribuibles según sexo en personas de 50 a 69 años, en Costa Rica desde 1990 al 2017 (Tasa por cada 100000 habitantes)

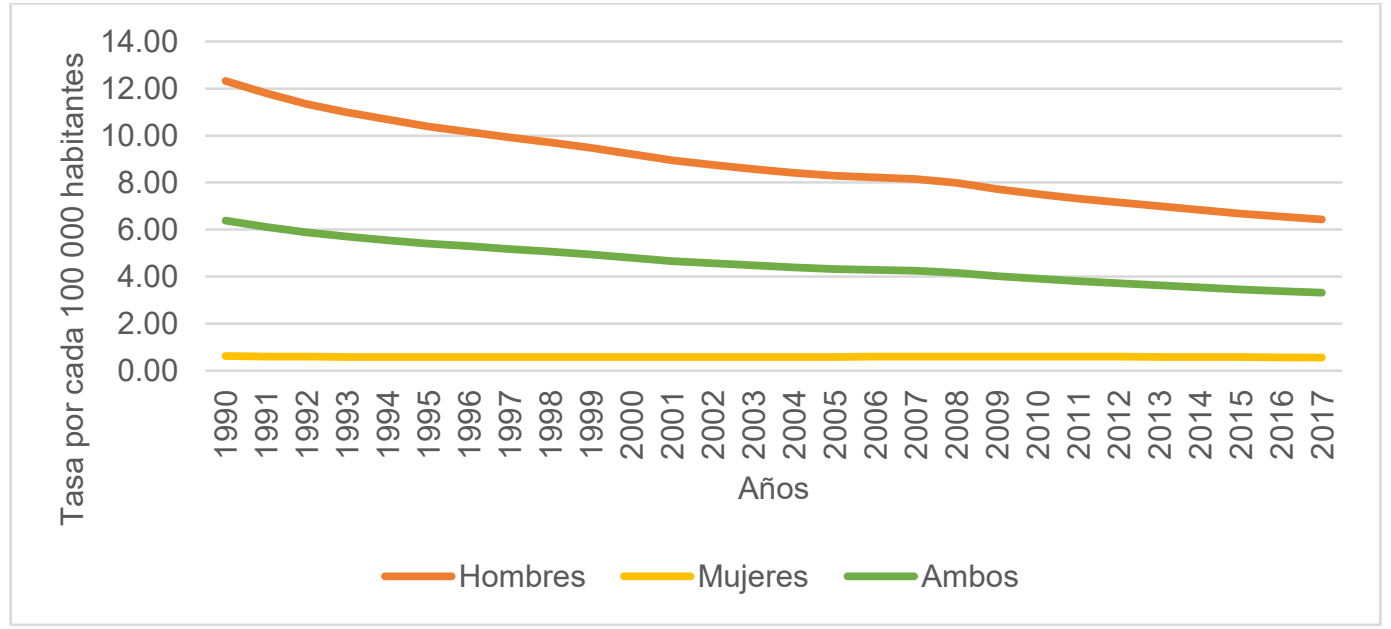

Fuente: Elaboración propia, con datos de GBD Compare | IHME Viz Hub [Internet]. [citado 19 de septiembre de 2021]. Disponible en: http://vizhub.healthdata.org/gbd-compare

La tasa de mortalidad que se muestra en la figura 2 se observa como la mayor tasa, la cual es de 12,32 por cada 100000 habitantes 
para el sexo masculino y la menor es de 6,43 .

Para el sexo femenino se observa que la mayor tasa de mortalidad es de 0,62 para 1990, y la menor de 0,56 para el 2017, no generando ningún impacto en la tendencia. Finalmente, para ambos sexos es mayor en
1990, con 6,38, y la menor de 3,32 para el 2017, observándose una disminución. Por lo tanto, la mayor tasa es para el sexo masculino con 12,32 en 1990 por cada 100000 habitantes, y la menor para el sexo femenino con 3,32 en el 2017.

Figura 3. Tasa de mortalidad por lesiones ocupacionales atribuibles según sexo en personas mayores de 70 años, en Costa Rica desde 1990 al 2017 (Tasa por cada 100000 habitantes)

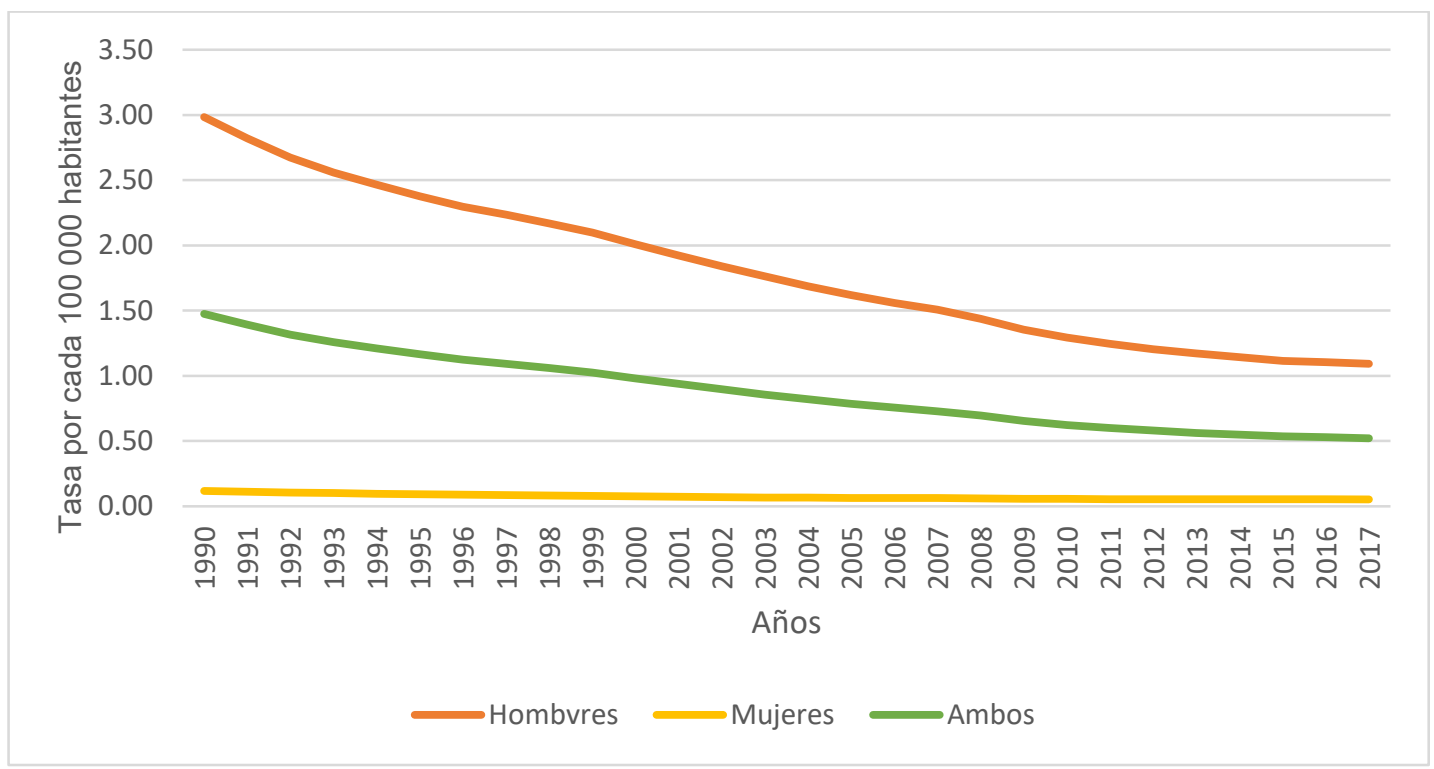

Fuente: Elaboración propia, con datos de GBD Compare | IHME Viz Hub [Internet]. [citado 19 de septiembre de 2021]. Disponible en: http://vizhub.healthdata.org/gbd-compare

\section{Tabla 1. Principales problemas de salud en las organizaciones}

Alcoholismo y dependencia química de drogas, medicamentos, tabaquismo, y otros.

SIDA: Ataca el sistema protector del organismo contra enfermedades.

Estrés en el trabajo.

Exposición a productos químicos peligrosos, como ácidos y asbestos, entre otros.

Exposición a condiciones ambientales frías, cálidas, contaminadas, secas, húmedas, ruidosas, poco iluminadas y otras.

Hábitos alimentarios inadecuados: obesidad o pérdida de peso.

Vida sedentaria sin contactos sociales, ni ejercicios físicos.

Automedicación sin cuidados médicos adecuados.

Fuente: Elaboración propia, con datos de Gómez L. Universidad de Costa Rica. :191. 
En la figura 3, para el grupo de mayores de 70 años, se observa cómo para el sexo masculino la tasa de mortalidad corresponde a 2,98 por cada 100000 habitantes, mientras que la menor es para el 2017 con 1,09 por cada 100000 habitantes; es notable cómo hay una marcada disminución, como se aprecia en los tres grupos estudiados anteriormente, pero la diferencia entre tasas es mínima. Finalmente, en la tasa de mortalidad para el sexo femenino también se observa una disminución de 0,07 , la cual no representa impacto alguno, observándose para 1990 una tasa de 0,12 por cada 100 000 habitantes, y para el 2017 es de 0,05, repitiéndose desde el 2013. Por lo tanto, la mayor tasa le corresponde al sexo masculino, con 2,98 en 1990, y la menor en 0.05 para el sexo femenino en el 2017.

\section{DISCUSIÓN}

La tasa de mortalidad fue mayor en 1990 por el grupo de 15 a 49 años en el sexo masculino. Como se puede ver en los 28 años en estudio, se logra observar un descenso al pasar los años bastante significativo, pero lamentablemente son los que predominan en la tasa de mortalidad por cada 100000 habitantes. Esto quiere decir que para el año en que se comienza el estudio fallecían 14,72 hombres por cada 100000 habitantes, y para el 2017 se redujo a 6,86 . Esto es positivo, pero la diferencia con el sexo femenino y con respecto al grupo de mayores de 70 año es exorbitante, debido que en 1990 fallecían 0.12 mujeres por cada 100000 habitantes, y ha ido en disminución hasta el 2017, lo que hace de nuevo llegar al tipo de funciones realizadas por las personas de acuerdo con el género y a la edad.

Aunque en las últimas décadas ha ido en gran descenso, es preocupante la cantidad de personas que fallecen por realizar trabajos peligrosos, o sin ningún tipo de supervisión o medida de seguridad que realmente se requiere. Como se ha observado en los indicadores epidemiológicos, es fácil observar una tendencia de cómo el sexo masculino es el grupo que corre más riesgo con respecto al femenino, y principalmente en el grupo de jóvenes y adultos, mientras que las mujeres aumentan con los AVISA y tienen una menor mortalidad, inclusive hasta un descenso hasta el mínimo, lo que cuestiona el tipo de funcionamientos que pueden realizar según el sexo y la edad, debido a que la mortalidad en los hombres mayores de 70 ha ido en un abrupto descenso, pero igualmente continúa ganando la tasa de mortalidad.

Es realmente preocupante cómo los hombres no cuentan con una buena calidad de vida en sus actividades laborales; se ha visto como los indicadores han ido en descenso, pero no en resolución de los problemas observados, por lo que se deberían plantear mejores opciones para lograr una mejoría en el menor tiempo posible.

Con respecto a los datos aportados por la OPS (9), aproximadamente el $65 \%$ de la población de América Latina y el Caribe forma parte de la fuerza laboral, asociado a que el trabajador promedio pasa $2 / 3$ de su vida trabajando; por lo que, a su vez, de acuerdo con los datos anteriores, se podría deducir que el ascenso de España y el descenso de Estados Unidos va a depender del país y cultura en la que se trabaja. Además, ocurren alrededor de 36 lesiones relacionadas con el trabajo por minuto, y 300 trabajadores mueren al día debido a lesiones ocupacionales, lo que indica que hay normas y regulaciones acerca de Salud Ocupacional, pero, aun así, hay un punto de quiebre, en el cual se podría trabajar mejor. Casi 800000 muertes por año ocurren por 
lesiones ocupacionales y 11000 por enfermedades ocupacionales a nivel mundial; esto lamentablemente no solo afecta a las empresas, sino que además es un gran gasto a nivel de los servicios de Salud, como se mencionó anteriormente, siendo el costo aproximadamente de las lesiones y muertes entre el 2 y el $14 \%$ del producto interno bruto en algunos países de esta región (9). Se logran mencionar, como principales problemas a nivel mundial, el dolor de espalda (37\%), la pérdida de la audición (16\%) y la EPOC (13\%); mientras que para América son hipoacusia ocupacional, intoxicaciones por plaguicidas, metales pesados, enfermedades respiratorias y asociadas a la piel. Cabe resaltar cómo, en América Central, se documentan 7000 casos de intoxicaciones agudas por plaguicidas por cada año, de acuerdo con el tipo de región en que se encuentran América Latina y el Caribe.

Estas comorbilidades mantienen un alto número de incidencia con respecto a las anteriormente mencionadas; sin embargo, no hay que dejar por fuera problemas que pudiesen llegar a ser letales, como lo son las intoxicaciones, las cuales constan de un número bastante significativo; por lo tanto, es imprescindible saber quiénes tienen la capacidad de tener acceso a servicios básicos de salud si algo sucediera, por lo que es preocupante que, según la OPS (9), alrededor del 10 al $15 \%$ de los trabajadores de América tienen este acceso, ya sea por descuido a de los patronos o la empresa, o simplemente por ser trabajadores informales. Dicho esto, es imperdonable que datos como los analizados anteriormente, no tengan el suficiente valor para que todos los trabajadores gocen de su derecho a la salud $y$, por lo tanto, se logre un $100 \%$ de cobertura a tener acceso a los servicios básicos de la salud.
Según el CSO, existen estadísticas de los siguientes años: 2013-2015-2016-2017, en las cuales existen registros acerca de la aplicación de los siguientes indicadores(10):

- Accidentados.

- No accidentados.

- Tasa de frecuencia.

- Tasa de gravedad.

- Días perdidos por año (por persona).

- Días perdidos por brote (epidemia, gripe o diarrea).

- Costos directos en que ha incurrido la empresa (incapacidades).

- Casos atendidos por el médico de la empresa en relación con el trabajo efectuado.

- Programas de vigilancia epidemiológica de acuerdo con los perfiles de riesgo.

De acuerdo con estos indicadores, para los años 2010-2013 se registraron (10):

2010: 1063

2011: 1372

2012: 1730

2013: 1758

Esto quiere decir que, con el pasar de los años, aumentaron los indicadores de riesgo laborales, por lo cual esto es negativo como parte de la salud pública del país, debido que son gastos que el INS debe reconocer.

En el mismo informe de los años posteriores (2015-2016-2017) (10-12), se observa cómo existe un aumento en la cantidad de personas aseguradas por riesgos de trabajo; para el 2015 estaban aseguradas 1298936 personas, para el 2016 se reportan 1296508 personas y para el 2017 se reportan 1 359916 personas. Por lo tanto, se observa cómo del 2015 al 2016 hubo una disminución de las personas aseguradas, pero para el 2017 existió un aumento de la 
cantidad de personas, inclusive para cualquiera de los años anteriores.

Para el reporte del 2016 se analiza el periodo comprendido desde el 2011 al 2016 sobre la siniestralidad laboral; esto quiere decir que existe un análisis anual sobre la cantidad de accidentes entre la población ocupada asalariada, excluyendo a los que no declaran en planilla o los que no fueron asegurados anteriormente a la ocurrencia del accidente. En el análisis se observa un aumento de la siniestralidad laboral, pasando del 7,9 \% para el 2011 y 2012, al $7,5 \%$ para el 2013 , seguido del $7,4 \%$ para el 2014 y posteriormente a un 7,5\% para el 2015 , aumentando de nuevo la incidencia al $7,8 \%$ para el 2016. Esto significa que los accidentes para personas asalariadas no van en descenso, sino que cursaron con una tendencia lineal; luego disminuyeron y para el 2017 aumentaron considerablemente.

Finalmente, para el 2017 se reporta una siniestralidad laboral de $8,4 \%$ (11). Con respecto a la información anterior, la incidencia anual ha aumentado en los últimos años; por lo que, según estos porcentajes, es importante crear alguna medida de información para trabajadores, con el fin de disminuir los accidentes laborales y, además, hay que tomar en consideración cómo, en el análisis expuesto, no toman en cuenta a trabajadores no asegurados, quienes sufren accidentes, y no se tiene realmente un resultado acerca de la incidencia de siniestralidad laboral a nivel nacional. En conclusión, se reportan, para el último año de esta investigación, las actividades y subactividades económicas con mayor índice ponderado de siniestralidad laboral, para el 2017 en Costa Rica (11), reportándose en primer lugar la agricultura con 3,60 , quien cuenta con las siguientes actividades: cultivo de caña de azúcar, cultivo de frutas tropicales y subtropicales; en segundo lugar se reporta la construcción con 3,59 , esto para quienes realizan construcción de edificios e instalaciones eléctricas y, en tercer y último lugar, el sector público con 1,80 , ya sea en las instituciones de salud y municipalidades, de acuerdo con las actividades peligrosas y riesgosas que realizan los trabajadores.

\section{CONCLUSIONES}

1. La tasa de mortalidad por lesiones ocupacionales en Costa Rica, se presentó a mayor escala en el sexo masculino entre 15 y 49 años, con la tasa de mortalidad más alta para el año 1990. En los siguientes años, esta tasa ha ido en descenso, pero siempre conservando el primer lugar. La menor tasa de mortalidad se presenta en el sexo femenino, en personas mayores de 70 años.

2. Alrededor del 10 al $15 \%$ de los trabajadores de América tienen este acceso a servicios básicos de la Salud en el ámbito laboral.

3. Los principales problemas a nivel mundial en el ámbito laboral son: dolor de espalda (37\%), pérdida de la audición (16\%) y EPOC (13\%).

4. Los principales problemas a nivel de América son: hipoacusia ocupacional, intoxicaciones por plaguicidas, metales pesados, enfermedades respiratorias y asociadas a la piel.

5. Se reportan 7000 casos de intoxicaciones agudas por plaguicidas por cada año en América Latina y el Caribe.

6. Ocurren alrededor de 36 lesiones relacionadas con el trabajo por minuto, y 300 trabajadores mueren al día debido a lesiones ocupacionales; además, 800000 muertes por año ocurren por lesiones ocupacionales, y 11000 por 
enfermedades ocupacionales a nivel mundial.

7. Las actividades y subactividades económicas con mayor índice ponderado de siniestralidad laboral para el 2017, en Costa Rica, son: primer lugar la agricultura con 3.60 para las siguientes actividades: cultivo de caña de azúcar, cultivo de frutas tropicales y subtropicales; en segundo lugar, la construcción con 3.59 para construcción de edificios e instalaciones eléctricas, y en tercer y último lugar, el sector público con 1.80 , ya sea en las instituciones de Salud y municipalidades.

8. En el 2017 se reporta una totalidad de 1 359916 personas aseguradas en el INS por riesgos de trabajo.

9. La siniestralidad laboral para el año 2017 fue del 8,4\%, aumentando notablemente durante los últimos 5 años.

\section{Los autores declaran no tener conflicto de interés.}

\section{REFERENCIAS}

1. T-UCSG-POS-MGSS-140.pdf [Internet]. [citado 22 de septiembre de 2021]. Disponible en: http://201.159.223.180/bitstream/3317/11089/1/T -UCSG-POS-MGSS-140.pdf

2. Toro Toro $\mathrm{J}$ de $\mathrm{L}$, Vega Falcón $\mathrm{V}$, Romero Fernández AJ, Toro Toro J de L, Vega Falcón V, Romero Fernández AJ. Los accidentes de trabajo y enfermedades profesionales y su aplicación en la justicia ordinaria. Rev Univ Soc. abril de 2021;13(2):357-62.

3. Padrón Vega $Y$, Moreno Pérez $S$ de las $N$, Márquez Ferrer A, González Valdés LM, Pérez Hernández F. Accidentalidad laboral en expuestos a riesgos biológicos en instituciones de salud. Rev Cienc Médicas Pinar Río. abril de 2017;21(2):529.

4. Rafael V. "Diseño de encapsulamiento del motor de una peletizadora de balanceado para reducir el ruido analizando materiales atenuantes en la empresa Exibal". 16 de junio de 2020 [citado 22 de septiembre de 2021]; Disponible en: http://dspace.unach.edu.ec/handle/51000/6647
5. Hernández-Sampieri R, Torres CPM. Metodología de la investigación. Vol. 4. McGraw-Hill Interamericana México^ eD. F DF; 2018.

6. Pinzón JED. Estimación de las tasas de mortalidad y letalidad por COVID-19 en Colombia. Rev Repert Med Cir. 3 de septiembre de 2020;8993.

7. GBD Compare | IHME Viz Hub [Internet]. [citado 19 de septiembre de 2021]. Disponible en: http://vizhub.healthdata.org/gbd-compare

8. Gómez L. Universidad de Costa Rica. :191.

9. Mitchell C, https://www.facebook.com/pahowho. OPS/OMS I Salud de los Trabajadores: Recursos [Internet]. Pan American Health Organization / World Health Organization. [citado 22 de octubre de 2019]. Disponible en: https://www.paho.org/hq/index.php?option=com content\&view=article\&id=1527: workers-healthresources\&ltemid $=1349 \&$ limitstart $=2 \&$ lang $=e$ S

10. Estadisticas Salud Ocupacional 2019.pdf [Internet]. [citado 23 de septiembre de 2021]. Disponible en: https://www.cso.go.cr/documentos relevantes/co nsultas/Estadisticas\%20Salud\%200cupacional\% 202019.pdf

11. Rica - ESTADÍSTICAS DE SALUD OCUPACIONAL.pdf [Internet]. [citado 19 de septiembre de 2021]. Disponible en: https://www.cso.go.cr/documentos relevantes/co nsultas/Estadisticas\%20Salud\%200cupacional\% 202017.pdf

12. Rica - ESTADÍSTICAS DE SALUD OCUPACIONAL.pdf [Internet]. [citado 19 de septiembre de 2021]. Disponible en: https://www.cso.go.cr/documentos relevantes/co nsultas/Estadisticas\%20CSO\%202016.pdf 